\title{
REFERRAL PATTERN OF PATIENTS RECEIVED AT THE NATIONAL REFERRAL HOSPITAL: CHALLENGES IN LOW INCOME COUNTRIES
}

\author{
Daudi O. Simba ${ }^{1}$, Naboth A.A. Mbembati ${ }^{2}$, Lawrence M Museru ${ }^{3}$, Leonard EK Lema ${ }^{2}$
}

\begin{abstract}
Objective: To examine the medical referral pattern of patients received at the Muhimbili National Hospital (MNH) in order to inform the process of strengthening the referral system.

Methods: The study design was a prospective study conducted at MNH during a 10-week study period from January to March 2004. The study sample consisted of patients referred to MNH

Results: Of the 11,412 patients seen, $72.5 \%$ were self-referrals. More than $70 \%$ of the patients seen required admission, though not necessarily at tertiary level. Only $0.8 \%$ came from outside the Dar es Salaam region. More than $70 \%$ of the patients seen required admission. Surgical services were required by $66.8 \%$ of patients, with obstetric conditions being most prominent $(24.6 \%$ of all patients). For those who were formally referred from other health services, lack of expertise and equipment were the most common reasons given for referral $(96.3 \%)$.

Conclusion: Efforts to improve referral systems in low-income countries require that the primary and secondary level hospitals services be strengthened and increased so as to limit inappropriate use of national referral hospitals.
\end{abstract}

Key words: Referral System, Tertiary Hospitals, Low- income countries, Tanzania

\section{Introduction}

Muhimbili National Hospital (MNH) is a University Teaching Hospital and national tertiary referral institution located in Dar es Salaam, Tanzania. The main responsibility of this hospital is to provide tertiary (Level 3) medical services for the whole country. However, $\mathrm{MNH}$ also offers medical care to the local urban population of 3 million people. Local services include secondary (Level 2) care for patients referred from any public or private health unit within Dar es Salaam, as well as primary (Level 1) care. Because $\mathrm{MNH}$ is a public institution funded solely by the government, it is perceived to be a cheap and convenient place to secure health care. This has led to overcrowding of patients in this hospital. The situation at $\mathrm{MNH}$ has been criticized for its use of overqualified staff and expensive facilities to provide Level 1 care in a tertiary health facility (1). The provision of primary care services in a tertiary institution is estimated to raise costs 6-fold (2). In addition, the quality of services is compromised, and access for patients who really need tertiary care becomes limited (3).

In response to these issues, and in accordance with the countrywide Health Sector Reforms that aim to strengthen tertiary level facilities, $\mathrm{MNH}$ is undertaking a major reform. This includes: (a) separation of the former Muhimbili Medical Centre into $\mathrm{MNH}$ and Muhimbili University of Health and Allied Sciences; (b) $\mathrm{MNH}$ administrative and financial management restructuring, with strengthening of general management, information technology, human resources and other support systems; and (c) major rehabilitation of in-patient units, assisted by funding from the Africa Development Bank. Changes to the in-patient units may involve reducing the current bed

Correspondence to: Dr. Daudi Simba, P. O. Box 65015, Dar es Salaam. E-mail: daudisimba@yahoo.com

${ }^{1}$ Department of Community Health, Muhimbili University of Health \& Allied Sciences (MUHAS), Tanzania, Dar es Salaam, Tanzania; ${ }^{2}$ Department of Surgery, MUHAS, ${ }^{3}$ Muhimbili Orthopaedic Institute, MUHAS capacity from 1500 beds to about 800 in order to provide higher quality care for a smaller but more complicated case mix.

In addition, $\mathrm{MNH}$ is undergoing an ambitious programme of modernization with financial support from the Abbott Fund, and management by the Axios Foundation. This has included the construction of a new 3storey outpatient treatment centre, increasing capacity to 1000 people per day. State-of-the-art laboratory equipment has been installed, resulting in one of the most advanced laboratories in Africa. Laboratory capacity for chemistry diagnostic tests has grown from 75 tests per day to up to 8000 tests per day. All donated laboratory equipment is maintained by field service engineers supported by the Abbott Fund.

The situation at the MNH is not unique, other tertiary care hospitals in Africa have been reported to face similar problems $(1,2,3)$. Attempts to streamline the referrals have been unsuccessful due to lack of sound research findings for guidance. This study was therefore conducted in order to examine the referral pattern of patients received at the $\mathrm{MNH}$ and some of the factors existing at the referring facilities that are likely to influence it with a view to informing the reform process.

\section{Materials and Methods}

\section{Design of the study}

A prospective study was done to determine the characteristics of patients received at $\mathrm{MNH}$. In addition, a cross-sectional study was done among referring health facilities in order to determine some factors likely to influence patient referral to $\mathrm{MNH}$.

\section{Sampling methods}

The study involved all patients seen at $\mathrm{MNH}$ from all sources over a period of 10 weeks from January to March 
2004. This included patients referred by health facilities and self-referrals. In addition, information was obtained from 29 referring health facilities in Dar es Salaam that were selected using convenient sampling using list of health care facilities provided by the City Medical Officer. The selected facilities included all 3 public hospitals, 5 of the 7 public health centres, 7 of the 56 public dispensaries, 6 of the 17 private hospitals, 2 of the 17 private health centres and 6 of the 347 private dispensaries.

\section{Research Instruments}

At the $\mathrm{MNH}$, a register was used to record patient information from daily recordings made at the Casualty and Medical Records Departments for all patients who arrived at $\mathrm{MNH}$ during the sample period, whether or not they had formal referral letters. Patient demographic characteristics, referral diagnoses, sources of referral and outcome were recorded.

At the referring health facilities, all patients referred to MNH during the study period were tracked using a temporary referral form in which patient demographic characteristics, referral diagnoses and reasons for referral were recorded. In addition, two checklists were used to obtain staff data, cadre and diagnostic and therapeutic procedures available at the referring health facilities. A semi-structured interview guide was prepared to gather information from referring clinicians about their reasons for referral.

\section{Data collection procedures}

Four medical officers and one assistant medical officer were recruited for data collection. The 5 research staff underwent a 2-day training session on the study methods and tools. The research instruments were pretested and refined before data collection commenced.

At the $\mathrm{MNH}$, one of the research staff was assigned to collect data from the Casualty and Medical Records Departments on a daily basis. Another of the research staff was responsible for compilation of the temporary referral forms brought in by arriving patients.

At the referring health facilities, the medical officers in charge were supplied with temporary referral forms to be completed by referring clinicians. On the first day of data collection the principal investigator met with all of the clinicians at the referring health facilities to explain the purpose of the study and how to complete the referral forms. Two research staff conducted the semi-structured interview to the clinicians who were responsible for referring patients from the 29 referring health facilities.

Ethical clearance was obtained from the Research and Publications Ethical Committee of the Muhimbili University of Health and Allied Sciences. Permission was sought from the heads of the respective institutions involved in the study. Individual consent was obtained from referring clinicians prior to interview. Quantitative data were coded and analyzed using the Epi-Info program (Version 6).

\section{Results}

\section{Patients' demographic characteristics}

During the 10-week study period, 12688 patients attended at $\mathrm{MNH}$ an average of 181 patients per day. Demographic data were available for 11909 patients. Of these, 7056 (59.2\%) were females and $48.8 \%$ were $20-39$ years of age.

\section{Source of referral}

A record of the source of referral was available for 11,412 patients seen at MNH during the study period. Selfreferrals accounted for most of the patients seen $(72.5 \%)$ while referrals from outside the region accounted for only $0.8 \%$ (Table 1). Public health facilities referred $22.4 \%$ of the patients seen, whereas private health facilities referred only $4.2 \%$. The proportion of patients received from other hospitals was $16.9 \%$, while $9.7 \%$ came directly from health centres or dispensaries.

Table 1. Distribution of patients by source of referral.

\begin{tabular}{lrrrrrr}
\hline \multicolumn{1}{c}{ Referral } & Public & \multicolumn{2}{l}{ Private } & Total & $\%$ \\
\hline \multicolumn{1}{c}{ Source } & Hospitals & $\begin{array}{c}\text { Primary } \\
\text { Health } \\
\text { Facilities }\end{array}$ & Hospitals & $\begin{array}{c}\text { Primary } \\
\text { Health } \\
\text { Facilities }\end{array}$ & & \\
\hline $\begin{array}{l}\text { Within Dar es } \\
\text { Salaam }\end{array}$ & 1898 & 665 & 38 & 442 & 3043 & 26.7 \\
$\begin{array}{l}\text { Outside Dar es } \\
\text { Salaam region }\end{array}$ & - & & - & - & 91 & 0.8 \\
Self & - & & - & - & 8278 & 72.5 \\
\hline Total & $1898(16.6 \%)$ & $665(5.8 \%)$ & $38(0.3 \%)$ & $442(3.9 \%)$ & 11412 & 100.0 \\
\hline
\end{tabular}

\section{Reasons for referral}

Clinicians in-charge of referring institutions were asked about the main reasons for referring patients to MNH. Nearly all of them (96.3\%) cited lack of expertise and lack of necessary equipment as the main reasons for referring the patients to $\mathrm{MNH}$. About half of the facilities reported lack of drugs (53.8\%) and space (50.0\%) as some of the important reasons for referral. Lack of affordability $(40.0 \%)$ was predominantly cited by private facilities.

\section{Presenting problems and diagnoses at $\mathrm{MNH}$}

Patients' presenting problems were classified according to specialty from which services were sought. Table 2 shows that surgical services, including orthopaedic and trauma accounted for the majority (36.8\%) of the patients seen at $\mathrm{MNH}$ followed by obstetric conditions $(24.6 \%)$, general medicine $(18.9 \%)$ and paediatric services $(9.4 \%)$ 
Table 2. Referral of patients by department/specialty

\begin{tabular}{lcc}
\hline & Number & \% of total \\
\hline Surgery & 4395 & 36.8 \\
Obstetrics & 2937 & 24.6 \\
General Medicine & 2248 & 18.8 \\
Paediatrics & 651 & 5.5 \\
Gynaecology & 632 & 5.3 \\
Neonatal & 469 & 3.9 \\
Psychiatric & 416 & 3.5 \\
Others & 183 & 1.5 \\
\hline Total & 11931 & 100.0 \\
\hline
\end{tabular}

Overall, $66.8 \%$ of patients required surgically oriented services at $\mathrm{MNH}$, while $31.8 \%$ required medically oriented services.

\section{Patient outcome at MNH}

Of the 10,735 patients whose records showed outcome at $\mathrm{MNH}, 70.1 \%$ required admission (Table 3). Admission rate was highest for patients from outside Dar es Salaam region $(84.7 \%)$, but admission rates were high even for self-referrals $(70 \%)$ and patients from within Dar es Salaam $(70 \%)$

Table 3. Patients referred to MNH by source of referral and outcome

\begin{tabular}{|c|c|c|c|c|}
\hline \multirow[t]{2}{*}{ Source of referral } & \multicolumn{4}{|c|}{ Patients outcome } \\
\hline & Admit & Discharge $^{a}$ & Re-direct ${ }^{\mathrm{b}}$ & Total \\
\hline Dar es Salaam region & $1984(70.0 \%)$ & $441(15.6 \%)$ & $408(14.4 \%)$ & $2833(25.8 \%$ \\
\hline Self-referral & $5473(70.0 \%)$ & $1600(20.5 \%)$ & $744(9.5 \%)$ & $7817(71.1 \%$ \\
\hline $\begin{array}{l}\text { Outside Dar es Salaam } \\
\text { region }\end{array}$ & $72(84.7 \%)$ & $5(5.9 \%)$ & $8(9.4 \%)$ & $85(0.8 \%)$ \\
\hline Total & $7529(70.1 \%)$ & $2246(19.1 \%)$ & $1160(10.8 \%)$ & $10735(100 \%$ \\
\hline
\end{tabular}

${ }^{\mathrm{a}}$ Discharge - patients treated at Casualty and allowed to go home

${ }^{\mathrm{b}}$ Redirect - patients referred from Casualty to outpatient clinics

\section{Discussion}

This study has shown that most of patients seen at $\mathrm{MNH}$ were self-referral $(72.5 \%)$ and that the majority $(70 \%)$ of these patients required admission, suggesting that they had genuine reasons for seeking health care. A previous study showed that up to $70 \%$ of patients admitted at $\mathrm{MNH}$ required Level 1 care. These findings indicate that a large proportion of the patients admitted at $\mathrm{MNH}$ are not appropriate. Similar findings have been reported in Zimbabwe where $60 \%$ of the patients admitted to the National Hospital were inappropriate for that level (2). A major consequence of this referral pattern is to impair the functions and quality of services offered by national referral hospitals in low-income countries.

The severe shortage of Level 1 care beds in Dar es Salaam is a major contributor to this situation. The city of Dar es Salaam has an estimated 0.17 to 0.25 Level 1 beds per 1000 population compared to a minimum requirement of 0.5 Level 1 beds per 1000 population in a metropolitan setting. The number of beds in Dar es Salaam compares less favourably with an average of 1.7 beds per 1000 reported in Lusaka, Zambia; approximately 10 times the capacity of the Dar es Salaam region (3).

The current population of Dar es Salaam is estimated to be 2.8 million (4). With only three Level 1 public district hospitals in the region, this corresponds to a ratio of one million people per hospital, which is five times the national recommendation for a district hospital. The high fees charged by private hospitals, ranging from Tsh 5000 to 10000 per day for a bed (equivalent to US\$5-10), are unaffordable to most of the people in a country where $35 \%$ live below the poverty line (5). The National Health Insurance Fund in Tanzania is still in its infancy and covers only government employees who are comparatively well off compared to an average Tanzanian. As a result, level 1 public facilities are overcrowded thus stretching resources beyond capacity, resulting in a lack of basic drugs, equipment and supplies. This acts as a disincentive to seek medical care in district hospitals and health centres, as reported elsewhere $(1,2)$.

Two-thirds $(66.8 \%)$ of the patients at $\mathrm{MNH}$ required surgically oriented services. This probably reflects the low output of Level 1 surgical care in district hospitals and the unaffordability of private hospitals. At $\mathrm{MNH}$, obstetric services received the highest proportion of referrals $(24.6 \%)$. This may have resulted from inadequate obstetric services in the district hospitals, and from a general lack of secondary level care in Dar es Salaam that could have provided for such services. Similar findings were reported in a study of referral systems in the Republic of Honduras, where obstetric services consumed the highest proportion $(21 \%)$ of available secondary care (6).

Fewer referrals were received for medically oriented services than for surgically oriented disciplines. This may be explained by medical problems being more readily taken care of by private facilities. A higher per-capita cost for surgical facilities, as compared to medical services might account for the relatively fewer private facilities offering surgical services in Dar es Salaam region.

Over half of the patients seen at tertiary hospitals in Nigeria came from the private sector (1). This is in contrast to our findings where despite having almost half of Level 1 beds in Dar es Salaam belonging to privately owned facilities, the proportion of patients referred from private hospitals to $\mathrm{MNH}$ was very low $(0.3 \%)$. A likely explanation for this finding is the tendency for private hospitals in Dar es Salaam to invite specialists from $\mathrm{MNH}$ to attend patients in their own hospitals, rather than referring the patients to $\mathrm{MNH}$.

During the period of this study, only $0.8 \%$ of the referrals were from outside Dar es Salaam region. This low figure might result from directives given to regional and district hospitals to seek tertiary care in other referral hospitals during the ongoing rehabilitation of the $\mathrm{MNH}$ infrastructure. Previous studies showed that $17 \%$ of outpatient referrals and $10 \%-15 \%$ of inpatients at $\mathrm{MNH}$ were from outside Dar es Salaam region. These proportions are somewhat low for a national referral hospital. Reports from other developing countries indicate that approximately $20 \%$ of patients attending tertiary 
hospitals came from outside the local city or region $(1,3)$.

In this study, data were collected for a period of 10 weeks which might not reflect the situation over the whole year. Retrospective studies have been reported to have the advantage of tracking referral patterns over longer periods, thus providing more comprehensive information $(2,6)$. However, attempts to gather retrospective data for this study failed due to insufficient records at both referring and receiving facilities.

\section{Conclusions}

Findings from this study highlight the difficulties faced by referral hospitals in low-income countries such as Tanzania in fulfilling their roles as a national referral hospital. Key factors impacting on patient health seeking behaviour were mainly the inadequacy of local district hospitals, and the absence of a secondary level facility in the region where the referral hospital is situated. Given the existing situation, strategies to restrict access to tertiary referrals, such as forbidding by-pass fees (1), are unlikely to be successful until adequate primary and secondary care have been provided. Thus, efforts to strengthen health care referral systems require, in addition to improving service capacity of the national hospitals, a corresponding effort to increase the capacity of primary and secondary level hospitals in the region. While this strategy will take a long time to be realized in many countries, short term strategies to increase access by the general population to the underutilized private sector need to be explored. Such strategies might include extension of national health insurance to cover all citizens.

\section{Acknowledgements}

We gratefully acknowledge funding support from the Axios Foundation. We thank the Muhimbili National Hospital, Muhimbili Orthopaedic Institute, Ocean Road Cancer Institute, City Medical Office and Muhimbili University of Health and Allied Sciences for their assistance in facilitating this study. The cooperation of staff at referring facilities was greatly appreciated.

\section{Conflict of Interest: None}

\section{References}

1. Akande TM. Referral system in Nigeria: Study of a tertiary health facility. Ann Afr Med 2004; 3:130-133

2. Sanders W, Kravitz J, Lewin S, McKee M. Zimbabwe's hospital referral system: does it work? Health Policy Plan 1998; 13:359-370

3. Blas $E$, Limbambala ME. The challenge of hospitals in the health sector reforms: the case of Zambia. Health Policy Plan 2001; 16(Suppl 2):29-43

4. National Bureau of Statistics. 2003. Tanzania Census 2002. Central Census Office, National Bureau of Statistics, Presidents Office, Planning and Privatization. Dar es Salaam. January 2003

5. National Bureau of Statistics 2002. National budget survey 2000/01. Dar es Salaam, July 2002. http://www.tanzania.go.tz/hbs/HomePage HBS.html Salaam, July 2002 .
[Accessed on 200708 30]

6. Omaha K, Melendez V, Uehara N, Ohi G. Study of a patient referral system in the Republic of Honduras. Health Policy Plan 1998; 13:433-445

Received 31 August 2007; Revised 12 February 2008; Accepted 20 February 2008 